\title{
Article \\ Emergent Space-Time in a Bubble Universe
}

\author{
James Moffat
}

check for updates

Citation: Moffat, J. Emergent Space-Time in a Bubble Universe. Symmetry 2021, 13, 729. https:// doi.org/10.3390/sym13040729

Academic Editor: Ignatios Antoniadis

Received: 9 March 2021

Accepted: 15 April 2021

Published: 20 April 2021

Publisher's Note: MDPI stays neutral with regard to jurisdictional claims in published maps and institutional affiliations.
Department of Physics, Kings College, University of Aberdeen, Aberdeen AB24 3FX, UK; jamesfostermoffat@gmail.com

\begin{abstract}
I exploit the close connection between the tessellation of space-time in the Regge calculus and an Eilenberg homology to investigate the deep quantum nature of space-time in a simple bubble universe of a size consistent with the Planck regime. Following the mathematics allows me to define this granulated space-time as the embedding space of the skeleton of a computational spin network inside a quantum computer. This approach can be regarded as a quantum simulation of the equivalent physics. I can, therefore, define a fundamental characterisation of any high-energy physical process at the Planck scale as equivalent to a quantum simulation inside a quantum computer.
\end{abstract}

Keywords: quantum gravity; quantum computing

\section{Introduction}

\subsection{Quantum Theory as a Black Box Model of Reality}

Quantum Theory is a guess at (or a prediction of) how an experiment at the quantum level relates inputs and outputs as real numbers shown on displays in the realm of classical mechanics. Good guesses are those which are consistent with all the existing experimental data (the facts) and have high predictive power, within their domain of application. Our currently accepted theory is the simplest of the good guesses, because the simplest explanation consistent with the facts is, we assume, nearest to the truth, (due to Occam's Razor). Finally, the more abstract the quantum theory, the more broadly relevant it is to the real world.

Quantum gravity is the quantum theory of space-time thus will have the following characteristics which I call Axioms.

Axiom 1. It will have an elegant and abstract mathematical structure, since this is the simplest way of expressing its logic (Occam's Razor again) and it should be widely applicable to the real world.

Axiom 2. It will be a theory based on the transformation of existing information (input values) into new information (the real or predicted output values).

Axiom 3. We require that for a given set of inputs, the outputs are correct (i.e., consistent with the facts), within its domain of application. The theoretical prediction correctly computes the output values but the mechanism by which this process is achieved is hidden from us. I call this quantum censorship.

Such a theory is a black box model of reality. We can predict the values of the output displays given the inputs, but we are forbidden, by quantum censorship, from seeing most of the connections inside the box.

An example of quantum censorship is the process called quantum tunnelling in the non-relativistic theory. This occurs for a particle trapped in a finite potential well (such as an atomic nucleus). The Born probability integrated over the part of the wave function lying outside the well gives the chance of finding the particle outside-but how did it get there? We are not allowed to even ask.

The mathematics of this black box theory of reality are best expressed in the language of linear operators as first pointed out by Born, Jordan and Heisenberg. This approach was initially systematised by Dirac and later by J von Neumann, see for example [1]. von 
Neumann's conceptual model of a * algebra (A *-algebra in this context is an algebraic ring of operators closed under the operator adjoint mapping $A \rightarrow A^{*}$. All algebras of operators mentioned in this paper are assumed to be ${ }^{*}$-algebras) (now called a von Neumann algebra) consisting of a ring (topologically closed for the ultraweak operator topology) of operators with an identity element acting on a Hilbert space still holds good. However, in an excellent discussion [2] Wald points out the clear advantages to thinking of this structure from the point of view of an abstract algebra A, closed in the ultraweak operator topology, with identity, called a $\mathrm{W}^{*}$-algebra [3] since the representation theory of $\mathbf{A}$ is then unbiased. I can then interpret any quantum theory (including quantum gravity) as follows: a quantum state $\rho$ is a positive element of the dual space $\mathbf{A}^{*}$ of $\mathbf{A}$ with $\rho(I)=1$. A representation $\pi$ is a homomorphism from $\mathbf{A}$ to a von Neumann subalgebra of the set of all bounded linear operators $\mathcal{B}(\mathcal{H})$ acting on a Hilbert space $\mathcal{H}$. The Hilbert space is either finite dimensional or separable. Each quantum state $\rho$ gives rise to a representation in which it is equivalent to a Dirac ket $\mid x>$ of the Hilbert space with norm one; this is the Gelfand-Naimark-Segal (GNS) construction [1]. An irreducible GNS representation corresponds to $\rho$ being a pure state. Since the state space is a weak*-convex, compact subset of the dual space, it is the closed convex hull of its extreme points These extreme points are the pure states. A finite system observable, such as the total system energy, corresponds to a bounded linear operator acting on this Hilbert space. The subset of states continuous for the ultraweak operator topology on $\mathbf{A}$ is the predual $\mathbf{A}_{*}$. The Gelfand transform mapping applied to an abstract $\mathrm{W}^{*}$-algebra $\mathbf{A}$ shows that $\mathbf{A}$ is (isomorphic to) the dual space of the linear hull of its predual, whch turns out to be a Banach space. More formally, we have

- An abstract $\mathrm{W}^{*}$-algebra $\mathbf{A}$ is a $\mathrm{C}^{*}$-algebra which is (isomorphic to) the dual space of a Banach space [3];

- Observations/measurements of a quantum system such as the total energy in a GNS representation correspond to discrete eigenvalues of the corresponding matrix operator. This implies that the set of observables corresponds to the subset of selfadjoint operators (with real eigenvalues).

\subsection{The Big Bang: Everything from Nothing?}

The evolution of space-time is governed by the laws of thermodynamics. The universe is the ultimate example of a closed system. Thus, the entropy of the universe increases over time from a low-entropy initial state [4]. This gives time its direction [5]. Data from the Planck satellite surveys of the Cosmic Microwave Background show that the Universe was logically simple near its beginning, which is compatible with having low entropy (a special baby). The Planck satellite data analysis implies that the power spectrum (the Fourier Transform of the spatial autocorrelation function) is close to scale invariant. Turok [6] argues on this basis for a simple beginning, which is in line with my axioms for quantum gravity. Within this context, I define a bubble universe to be a possibly unique simple bubble of space-time developing within the Planck regime over a few atto-seconds after the big bang.

\subsection{Gravity}

In the pre-quantum world, gravity is a classical theory of space-time as a smooth curved manifold, with the Ricci measure of local curvature equal to the local system mass-energy (neglecting constants). This corresponds to equating two covariant tensors $R_{\mu v}=E_{\mu v}$ of equal rank. This in turn, following the mathematics of Riemann, makes the subsequent expression covariant relative to a change of generalised coordinate systems (thus known as General Relativity or GR). Einstein's non-quantum theory of gravity (GR) is very dependent on general covariance [7]. This theory is not consistent with Occam's Razor; it brings too much baggage which must be cut away. For example, at the beginning of the big bang, Einstein's theory implies that the curvature of the universe and the energy density are both infinite, which makes no sense. It is also impossible to measure intervals in space smaller than the Planck length using a standard measuring rod (such 
as a laser beam and a mirror). To make the measurement requires inserting at least one wavelength of monochromatic light into such an interval. The energy of such a photon $E=h v=\frac{h c}{\lambda}>\frac{h c}{l_{p}}$ which is extremely high, leading to local collapse into a black hole. Attempting to discriminate further by increasing the energy of the photon, simply increases the size of the black hole. There are more problems; creating two such black holes which are entangled leads to a wormhole in our 'apple' of space-time. This comes from the thought experiments of Maldacena and Susskind (Known as 'ER =EPR').

A simpler quantum-based theory, based indeed on the elegant idea of small finite increments of space-time, is adequate to the task, as we will show. The point of these quantum assumptions is to impose a granularity on space-time at the Planck scale; as is implied by most theories of quantum gravity; see for example [8] (Chapter 2). This corresponds to a lower limit to measurement and a finite theory in the sense of Dirac. For a closed and bounded (compact) subset of a granular space-time, the vacuum energy is always finite. A renormalisation which is based on convergence of neighbouring events, has a lower limit at the Planck scale.

We take forward the fusion of space and time into a single entity and the representation of gravity as a set of oriented, locally bounded, compact double-cones. The discrete nature of a computational spin network $X$ implies that only at most a countable number of these double cones are required; each one attached to an embedded node of $X$.

These act like waymarks, directing the local discrete movements through the embedded network embdX from node to node by the action of the translation subgroup of the relativistic Poincare group [8] (Chapter 2). From this perspective, flat space-time is a very special case in which each of the local double-cones has the exact same orientation as its neighbours. Thus, the existence of a gravitational field is the generic case. This is similar to one of the key ideas behind special relativity (The other being the invariance of the speed of light in vacuo): that a moving frame of reference is the generic case, not requiring further justification.

\section{Computational Spin Networks}

Consider now the dynamics of the Dirac quantum state of a spin $\frac{1}{2}$ fermion in a way which is consistent with the requirements of special relativity. This quantum state is four dimensional, with the first two components' corresponding to the particle and the second two components corresponding to the anti-particle, each corresponding to a Weyl 2-spinor. With planar isotopy, it is possible to associate various locally deformable lines in the plane with Weyl 2-spinor calculations, giving rise to topological structures called spin networks. The motivation for Penrose in developing spin network theory was as part of his belief that discreteness was a fundamental part of a unification of general relativity and Quantum theory. Penrose's approach has links to the Jones polynomials of knot theory (a favourite of E. Witten) via the Reidemeister moves which connect two isomorphic 3-dimensional knots.

I exploit such network ideas, together with the work of T. Regge on tessellation limits as 4-dimensional manifolds in general relativity [9-11] which I reimagine. General relativity is now the 'poor relation', as a mere approximation to a quantum tessellated space-time at the Planck scale. I also note that the dynamics of a tessellated space-time are the limit of the smooth system dynamics $[10,11]$.

I define a computational spin network to be a finite or countable quiver consisting of a directed graph, where the nodes represent entangled spin inputs, and a directed random link between two nodes corresponds to a quantum gate such as the Controlled-NOT Gate, with entangled state inputs. I assume in what follows that the number of nodes $n$ is finite.

Given two input qubit quantum states the basic NOT quantum gate corresponds to a $2 \times 2$ unitary matrix with $U|1>=| 0>$, etc. The Hadamard quantum gate rotates each such spin eigenvector into a quantum superposition. All quantum gates in a quantum computer correspond in this way to multiplying the input state vector by a unitary matrix mapping, usually of the form $S U(2)$ when considering just qubits. In the following we denote the joint tensor product state of $|x\rangle$ and $|y\rangle$ as $|x y\rangle$. 
I can now perform a set of steps from one node to the next neighbouring node through the network, and we can interpret this process in physics terms as a set of transitions, such as $\left|y_{2}>\rightarrow\right| y_{3}>$ as steps across our computational spin network. We need to show that the initial state $\left|y_{0}\right\rangle$ is retrievable from the end state $\left|y_{3}\right\rangle$. For this last part, we ask our avatar scientists Alice and Bethe to help. Assume that Alice prepared both the network and the initial quantum state $\mid y_{0}>$ and wishes to send this state to Bethe. She constructs the pathway $\left|y_{0}\right\rangle \rightarrow\left|y_{1}>\rightarrow\right| y_{2}>\rightarrow \mid y_{3}>$ ending at Bethe. She then performs a measurement across the tensor product eigenstates which form the basis states (as shown in [8]) $|00>;| 10>; \mid 01>$ and $\mid 11>$. She texts the result to Bethe as '00', '10', '01' or ' 11 '. Bethe then applies the appropriate $2 \times 2$ unitary matrix to the state supplied by the network to turn it into the state $\left|y_{0}\right\rangle$. For example, suppose the text message '01' is sent, travelling at the speed of light. When Bethe receives the message, she applies a matrix such as $U(01)^{-1}=U(01)=\sigma^{1}$, one of the Pauli spin matrices to convert her 'travelled' state back to the original state. This general process is called quantum teleportation and forms a key part of quantum computing.

\section{The Homology Invariants of Space-Time}

I now give a proof of the result (similar in spirit to Maldacena's musings on the connection between a quantum field and an anti-de Sitter space) that this transport from node to node of the computational spin network is equivalent to the action of a sequence of discrete relativistic translations of space-time from one node to the next neighbouring node.

\section{Homology Invariants of the Network Structure}

Homology is concerned with the classification of topological spaces through decomposition of the space into geometric objects such as triangles, which tesselate the surface of the structure, just as in the Regge theory. In Loop Quantum Gravity, this is closely related to Rovelli's idea of an area operator whose eigenvalues correspond to the discrete measurement of area. For both these reasons we choose to focus on the homological invariants of a computational spin network. From this analysis we will show that the 'machinery' of such algebraic approaches is fully available to us at this fundamental network level. It allows us to develop the desired mathematical linkage between transport across nodes of this spin network and discrete transitions in embedded space-time [8] (Chapter 2). The contravariant dual to a homology is a cohomology. In a similar way the Gromov-Witten invariants of string theory $[12,13]$ exploit this machinery to provide a topologically based classification of the landscape of Calabi-Yau topological manifolds.

Network nodes represent quantum computational gates. Following a suggestion of references [14,15], I assume that the network topology is that of an evolving random network, of class $(n, p r)$ derived from a fixed probability $p r$ of a link between two randomly chosen nodes.

I now consider a computational spin network $\boldsymbol{X}$ defined as a finite quiver consisting of a directed graph with a set of nodes $n$ corresponding to entangled spin inputs and directed links between these nodes, with an upper bound of $\frac{n(n-1)}{2}$ links. Percolation theory [16] applied to this structure implies a critical value of $p r$ denoted $p_{c}$ for which the network becomes path connected. The value $p r=1$ corresponds to a fully connected graph.

In the usual way, I can consider this graph as the skeleton of a simplicial tessellated surface when embedded in a dimensioned topological space. For $p_{c}<p r \leq 1$ we have a range of units of tessellation, including 3-simplexes (tetrahedra) and potentially higher dimensional generalisations. We can use these basic properties to develop the structures, including simplicial complexes, of an Eilenberg singular homology $[17,18]$ associated with a given computational spin network. I cannot in principle resolve the embedding space into minimal subunits of space-time smaller than triangles (2-simplexes) with finite 1dimensional edges. I define such a topological space as a discrete embedding space. This is consistent with increments of space and time which can be traded off against each other 
as part of a more fundamental unit; the space-time vector forming the 1-dimensional boundaries of a triangular 2- simplex.

Given a dimensioned embedding of $X$, we assume that the resultant topological space $e m b d X$ is both finite dimensional and discrete in the sense discussed above. For the set of $p$-simplexes $\Delta_{i}^{p}$ forming part of the structure of our abstract computational network, we can then define a set of embedding functions $\left\{e_{i}^{p}: \Delta_{i}^{p} \rightarrow \operatorname{embdX} ; p \geq 2\right\}$. The $p$-simplex $\Delta_{i}^{p}$ has $p+1$ boundary faces, each forming a $(p-1)$-simplex; and each of these has a lift function $f_{i j}^{p-1}: \Delta_{i j}^{p-1} \rightarrow \Delta_{i}^{p} \circ f_{i j}^{p-1}: \Delta_{i j}^{p-1} \rightarrow e m b d X$.

I define space-time in the Planck regime as this unique geometric embedding of the singular homology induced by the computational spin network.

I now define, following the math, the boundary function $\partial_{p}$, for $p \geq 2$, acting on the embedded $p$-simplex $\Delta_{i}^{p}$ in terms of its $p+1$ boundary surfaces as the alternating sum of the boundary face embedding functions

$$
\partial_{p} \circ e_{i}^{p}=\sum_{j=0}^{j=p}(-1)^{j} e_{i}^{p} \circ f_{i j}^{p-1}
$$

The alternating sum definition ensures that the boundary of a boundary is trivial. This enables the creation of the chain complex, defining the pth-homology module $H_{p}$ as

$$
H_{p}=\frac{\operatorname{Ker} \partial_{p}}{\operatorname{Im} \partial_{p+1}}
$$

If $X$ and $Y$ are elements of the same topological equivalence class, there is a homeomorphism $\varphi: X \rightarrow Y$, with an inverse $\sigma=\varphi^{-1}: Y \rightarrow X$. The boundary function on $Y$ is given by;

$$
\hat{\partial_{p}}=\partial_{p} \circ \sigma=\sum_{j=0}^{j=p}(-1)^{j} f_{i j}^{p-1} \circ \sigma
$$

We now follow the Alexander-Kolmogorov (A-K) construct for a cohomology dual to this homology. The topological invariant $C_{p}$ consisting of elements of the form $n_{i} e_{i}^{p}$ is the freely generated abelian group on the set of all $p$-embeddings $e_{i}^{p}$. Define $\operatorname{HOM}\left(C_{p}, T\right)$ to be the set of all homomorphisms $\varphi: C_{p} \rightarrow T$ where $T$ is the abelian translational subgroup of the relativistic Poincare group. Following the A-K construct, $\operatorname{HOM}(*, T)$ is a contravariant functor transforming the homology chain complex into a cohomology chain complex. For if $\varphi \in \operatorname{HOM}\left(C_{p-1}, T\right)$, then $\partial^{p}(\varphi) \equiv \varphi \circ \partial_{p} \in \operatorname{HOM}\left(C_{p}, T\right)$.

Identifying embdX as tessellated space-time, let $S$ be a step across the computational spin network from one node to another. $S$ is the vector sum of a set of minimal steps from a node to some neighbouring node of the random network. Each such minimal step $\mathrm{s}$ is a translation $t$ from node $n(k)$ to the node $n(l)$ along the boundary edge of a $p$-simplex $\Delta_{i}^{p}$ for some value of $p \geq 2$. Thus, we can choose a local homomorphism $\varphi$ in the cochain complex $\operatorname{HOM}\left(C_{p-1}, T\right)$ with $\varphi(s)=t$, with $t$ an element of the relativistic abelian translational group T. Gluing these local homomorphisms together gives us the desired mapping.

This result implies a fundamental characterisation of any high-energy physical process at the Planck scale as equivalent to a quantum simulation inside a quantum computer.

A discrete path in space-time at the Planck scale, as we have so far generated it, can be considered as a set of linked causally directed vectors in the embedding space of the skeleton of an Eilenberg homology. Even more formally, we define the path as a series of $n$ linked increments $\mathbf{a}(j)$ each with varying direction within their local forward light cones, such that the path begins at $\mathbf{x}(\mathbf{0})$ and ends at $\mathbf{x}(\mathbf{1})$, with $T(a(j)): x \rightarrow x+a(j)$ elements of the relativistic translation subgroup $T$. The total path is then generated by the ordered product $\prod_{j=1}^{j=n} T(a(j)) \boldsymbol{x}(\mathbf{0})$ with the final end point $\mathbf{x}(1)=x(\mathbf{0})+\sum_{j=1}^{j=n} a(j)$. For a fixed initial point $x(0)$ we can identify this path with the finite group product $\prod_{j=1}^{j=n} T(a(j)) \in \mathbf{T}$. 
If all the increments point in the same direction, and the local light cones have the same orientation, one to another, this gives a straight path in space-time. If, however, the local light cones are rotated one to another, then the path will be piecewise curved. The choice of settings for the light cones constrains the trajectory of the resultant path in the same way that the gravitational field in Einstein's theory constrains a geodesic path between two space-time events to be of the form

$$
\frac{d^{2} x_{\tau}}{d s^{2}}+\Gamma_{\mu \nu}^{\tau} \frac{d x_{\mu}}{d s} \frac{d x_{v}}{d s}=0
$$

where, in Christoffel notation, $\Gamma_{\mu v}^{\tau}=\{\mu v, \tau\}$, the Christoffel symbol. For the quantum case a reorientation of the locally compact light cones, resulting in a different curvature, corresponds to an isomorphism between a path and a finite set of such orientations, one for each node of our network.

The orientation of these locally compact light cones in classical space-time corresponds to the definition of three angles; pitch, roll and yaw $(\theta, \varphi, \rho)$, as a function of time $t$. We can capture these parameters by the general quaternion $x=t+i \theta+j \varphi+k \rho$. For the quantum case we can write this using the Dirac quantum quaternion matrices (so-called because they satisfy the axioms of a Clifford algebra).

\section{Discussion and Conclusions}

I start this discussion from the reasonable assumption that space-time is granular in a bubble universe of a size consistent with the Planck regime. From this beginning many destinations are possible, including the Jones polynomials of knot theory. I focus in this paper on the close relationship between a Regge tessellated space-time and an Eilenberg homology allows me to define granulated space-time as the embedding space of the skeleton of a computational spin network inside a quantum computer. This leads me to the following conclusion, by following the math. I can define a fundamental characterisation of any high-energy physical process at the Planck scale as equivalent to a quantum simulation inside a quantum computer.

Funding: This research received no external funding.

Institutional Review Board Statement: Not applicable.

Informed Consent Statement: Not applicable.

Data Availability Statement: Not applicable.

Conflicts of Interest: The author declares no conflict of interest.

\section{References}

1. Kadison, R.; Ringrose, J. Fundamentals of the Theory of Operator Algebras; Academic Press Ltd.: London, UK, 1983.

2. Wald, R. Quantum Field Theory in Curved Spacetime and Black Hole Thermodynamics; University of Chicago Press: Chicago, IL, USA, 1994.

3. Sakai, S. C $C^{*}$-Algebras and $W^{*}$-Algebras; Springer: Berlin, Germany, 1971.

4. Penrose, R. The Road to Reality; Random House: London, UK, 2004.

5. Davies, P. The Physics of Time Asymmetry; University of California Press: Berkeley, CA, USA, 1977.

6. Turok, N. Available online: https://www.imperial.ac.uk/news/186589/the-universe-incredibly-simple-says-renowned/ (accessed on 31 March 2021).

7. Petruzziello, L. A dissertation on General Covariance and its application in particle physics. J. Phys. Conf. Ser. 2020, 1612, 012021. [CrossRef]

8. Moffat, J. A Mathematically Coherent Quantum Gravity; Institute of Physics: London, UK, 2020.

9. Regge, T. General relativity without coordinates. Nuovo Cim. 1961, 19, 558-571. [CrossRef]

10. Regge, T.; Willams, R. Discrete structures in gravity. J. Math. Phys. 2000, 41, 3964-3984. [CrossRef]

11. Barrett, J. The geometry of classical Regge calculus. Class. Quantum Grav. 1987, 4, 1565-1576. [CrossRef]

12. Knezevich, M.; Manin, Y. Gromov-Witten classes, quantum cohomology, and enumerative geometry. Comm. Math. Phys. 1994, $164,525-562$. 
13. Li, A.; Ruan, Y. Symplectic surgery and Gromov-Witten invariants of Calabi-Yau 3-folds. Invent. Math. 2001, 145, 151-218. [CrossRef]

14. Requardt, M. Space-time as an order-parameter manifold in random networks and the emergence of physical points. Quantum Theory Symmetries 1999, 2000, 555-561.

15. Smolin, L. An Invitation to Loop Quantum Gravity. (hep-th). Available online: https://arxiv.org/abs/hep-th/0408048 (accessed on 20 March 2021).

16. Moffat, J. Complexity Theory and Network Centric Warfare; Diane Publishing: Darby, PA, USA, 2010.

17. Eilenberg, S.; Steenrod, N. Foundations of Algebraic Topology; Princeton University Press: Princeton, NJ, USA, 2015.

18. Nunke, R.; Rotman, J. Singular cohomology groups. J. Lond. Math. Soc. 1962, 1, 301-306. [CrossRef] 